\title{
An Active Noise Control Algorithm Principle and Analysis without Secondary Path Identification Based on Kalman Filter
}

\author{
Can Chen ${ }^{1,2}$, Guo Wei ${ }^{2}$, Ru He ${ }^{3}$ \\ ${ }^{1}$ Computer \& Information Science Department, Delaware University , \\ Newark, DE 19716, USA \\ ${ }^{2}$ Automatic Testing \& Control Department,Harbin Institute of Technology, \\ Harbin , 15001, China \\ ${ }^{3}$ Earth Science College, Northeast Petroleum University, Daqing 163318, \\ China
}

\begin{abstract}
Most active noise control (ANC) algorithms require the identification of the secondary path, thus suffer from large complexity, increased residual noise power and algorithm divergence. In this paper, we propose a novel ANC algorithm without secondary path identification based on Kalman filter, referred to as Model Error Compensatory Kalman Filter (MECKF). The ANC problem is described in discrete-time state-space form first, then the dynamics of the primary path can be attributed to the state variables. Kalman filter is applied to estimate the weights using residual noise sequence. Furthermore, based on acoustics properties and stochastic theory, we introduce a model error compensating mechanism by shifting the influence of the unknown secondary path into variance of measurement matrix. In addition, a new method of estimating the statistical properties of the noise in dynamic model is given in the context of ANC system, with merits of reduced computational complexity, increased convergence rate, and ensured real-time.

Keywords: active noise control (ANC),Kalman filter, secondary path, state-space.
\end{abstract}

\section{Introduction}

Active noise control (ANC) works on the principle of destructive interference between the sound fields generated by original "primary" sound source and that by other "secondary" sources, which has become an important area in noise control research, due to its effectiveness in low-frequency noise cancellation. Adaptive ANC has been well-established with the development of adaptive signal processing, it is essential that adaptive ANC algorithm--filtered-x algorithm requires identification of the secondary path. Most linear ANC system use the Least Mean Square (LMS) [1] algorithm due to its simplicity. To overcome the 
slow convergence and high sensitivity to the eigenvalue spread [2-3] problems associated with LMS, Recursive Least Square (RLS) [2] algorithm is often used. Their poor performance in unstable environment lead to the combination between Kalman filter [4-7] and ANC, which is a general framework of RLS algorithm, the demand of small number of taps can reduce its high computational complexity [3], also the Kalman filter may be easily applied in ANC by today's DSPs. Paulo Lopes [4] fitted a specialized version of the Kalman filter to both control filter adaptation and secondary path modeling. The fast-array Kalman filter proposed by Fraanje Rufus [5] has a fast convergence rate. Bambang Riyanto [6] developed a learning algorithm for diagonal recurrent neural networks based on Kalman filter referred to as Diagonal Recurrent Extended Kalman Filter.

Since the requirement of identifying the secondary path caused several problems: increase the complexity and residual noise power of the control system, error in identifying the secondary path may cause algorithm to diverge, a control algorithm that does not require secondary path identification has become popular currently [8-12]. Of all these method, the most attracting one is the Direction Search Least Mean Square (DSLMS) proposed by Zhou [8], which is based on geometric analyses and the strict positive real (SPR) property of the filtered-x LMS, simple to implement and yields good performance, however, converges slowly and will diverge at a particular frequency band in which the secondary path phase response near or equal to $\pm 90^{\circ}$.

Because require prior knowledge of the model and the statistical properties of model noises, Kalman filter now is constrained within filtered-x ANC algorithm. Here, for the first time, we introduce a Kalman filter based ANC algorithm without secondary path identification, Model Error Compensatory Kalman Filter (MECKF) algorithm. In state interpretation of feedforward ANC system, our proposed method formulates controller weight estimation problem without secondary path identification as Kalman filter parameter estimation, adjusting Kalman gain to compensate the unknown secondary path effect, meanwhile a new method to determine model noise is given. The new algorithm enjoys fast convergence, low residual noise power, good performance in both stationary and non-stationary environment.

\section{Algorithm principle and implication}

It is critical in ANC algorithm without secondary path identification to estimate controller weight and compensate the unknown secondary path influence.

\subsection{State-space form of ANC system}

The general block diagram of feedforward ANC problem is shown in Fig.1. $P(z), S(z)$ and $W(z)$ represent the primary path, secondary path and ANC controller, respectively; $r(n)$ is the original disturbance signal and also the input of the adaptive algorithm, which becomes primary signal $d(n)$ propagating through primary path; $v(n)$ and $e(n)$ are additive noise and residual noise, 
respectively; $u(n)$ is the control signal output by ANC controller; $\hat{y}(n)$ is the secondary signal. The most common form of $W(z)$ is the transverse FIR adaptive filter.

$$
W(z)=w_{0}+w_{1} z^{-1}+\cdots+w_{M-1} z^{-M+1}
$$

Where, $M$ is the tap number of the controller, $w_{i}(i=0,1, \cdots, M-1)$ is the weight of the controller. Weight vector is $\mathbf{w}=\left[\begin{array}{llll}w_{0} & w_{1} & \cdots & w_{M-1}\end{array}\right]^{\mathrm{T}}$

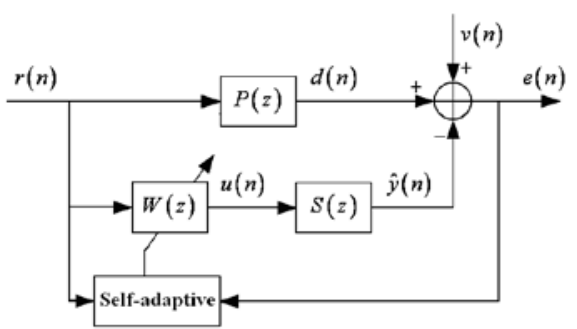

Fig.1 General block diagram for an active noise control (ANC) problem

The objective of ANC problem is to seek the optimal weight vector $\mathbf{w}^{O}$, with which the optimal controller $W^{O}(z)$ can generate a control signal $u(n)$, such that the secondary signal $\hat{y}(n)$ cancels the sum of primary signal $d(n)$ and additive noise $v(n)$. Namely, a series connection of the optimal controller and secondary path is the best approximation of the primary path, $P(z)=W^{O}(z) S(z)$. Then the dynamics of the primary path accounts for weight estimation errors. The weight vector is updated by (2).

$$
\mathbf{w}(n)=\lambda \mathbf{w}(n-1)+\mathbf{v}_{1}(n)
$$

Where $\mathbf{v}_{1}(n)$ is the estimation noise, $\lambda(0<<\lambda \leq 1)$ is the factor of weight adapting.

Let the reference signal vector $\mathbf{r}(n)=\left[\begin{array}{llll}r(n) & r(n-1) & \cdots & r(n-M+1)\end{array}\right]$

Then the secondary signal is

$$
\hat{y}(n)=[\mathbf{r}(n) \mathbf{w}(n)] * S(n)
$$

Where, $S(n)$ represents the secondary path in discrete-time domain. Define $y(n)=d(n)+v(n)$, we can write

$$
y(n)=[\mathbf{r}(n) \mathbf{w}(n)] * S(n)+e(n)
$$

Where, * represents convolution.

According to the state-space model of discrete linear dynamic system in control theory, (2) is the state equation of ANC system, (4) is the measurement equation. Thus, we can update weights by using state estimation solution.

\subsection{Kalman filter weight estimation}

Since the Kalman filter provides a minimum variance estimate of the state based on minimum mean square error, with fast convergence and high performance in both stationary and non-stationary environment, we use this filter to estimate 
$\mathbf{w}(n)$.

We modeled secondary path as FIR filter with coefficients as

$$
\mathbf{S}(n)=\left[S_{0}, S_{1}, \cdots, S_{M_{S}-1}\right]^{T}
$$

Assuming $\mathbf{v}_{1}(n)$ to be a zero-mean white noise, weight updating becomes

$$
\mathbf{w}(n)=\lambda^{i} \mathbf{w}(n-i)
$$

Then (4) becomes

$$
y(n)=\mathbf{r}_{S \lambda}(n) \mathbf{w}(n)+e(n)
$$

Where

$$
\mathbf{r}_{S \lambda}(n)=\left(\sum_{i=0}^{M_{S}-1} \mathbf{r}(n-i) \lambda^{-i} S_{i}\right)
$$

Based on the definitions in state-space, (2) and (7) are rewritten as

$$
\begin{aligned}
& \mathbf{w}(n)=\mathbf{F} \mathbf{w}(n-1)+\mathbf{v}_{1}(n-1) \\
& y(n)=\mathbf{r}_{s \lambda}(n) \mathbf{w}(n)+v_{2}(n)
\end{aligned}
$$

Where, $\mathbf{w}(n)$ is $L \times 1$ weight vector; $y(n)$ is measurement vector; $\mathbf{F}=\lambda \mathbf{I}$ ( $\mathbf{I}$ is $L$ order unit matrix) is state transition matrix (STM); $\mathbf{r}_{S \lambda}(n)$ is $1 \times L$ measurement sensitivity matrix; vector $\mathbf{v}_{1}(n)$ is $L \times L$ system noise, $v_{2}(n)$ is $1 \times 1$ measurement noise, with statistical properties as follows

$$
\begin{array}{ll}
\mathrm{E}\left[\mathbf{v}_{1}(n)\right]=\overline{\mathbf{v}}_{1}(n), & \operatorname{cov}\left[\mathbf{v}_{1}(n), \mathbf{v}_{1}(m)\right]=\mathbf{Q}_{1}(n) \delta(n-m) \\
\mathrm{E}\left[v_{2}(n)\right]=\bar{v}_{2}(n), & \operatorname{cov}\left[v_{2}(n), v_{2}(m)\right]=Q_{2}(n) \delta(n-m) \\
\operatorname{cov}\left[\mathbf{v}_{1}(n), \mathbf{v}_{2}(n)\right]=\mathbf{0} &
\end{array}
$$

Where, $\mathbf{Q}_{1}(n)$ is $L \times L$ system noise variance matrix, $Q_{2}(n)$ is measurement noise variance matrix, $\delta(n-m)$ represents Kronecker function.

Applying Kalman filter to search $\mathbf{w}^{O}$ can be describe as: seek the optimal estimation (the linear minimum variance estimation) of $\mathbf{w}(n)$ based on measurement data $y(n)$, makes estimation $\hat{y}(n \mid n-1)$ approximate to $y(n)$, which means $e(n)=y(n)-\hat{y}(n \mid n-1)=\min$. The cost function of the linear minimum variance estimation is given by

$$
\mathbf{J}=\mathrm{E}\left[(\mathbf{w}(n)-\hat{\mathbf{w}}(n \mid n-1))^{T}(\mathbf{w}(n)-\hat{\mathbf{w}}(n \mid n-1))\right]
$$

Filter state-error and its covariance are defined as

$$
\begin{gathered}
\tilde{\mathbf{w}}(n \mid n)=\mathbf{w}(n)-\hat{\mathbf{w}}(n \mid n) \\
\mathbf{K}(n \mid n)=\mathrm{E}\left[\tilde{\mathbf{w}}(n \mid n) \tilde{\mathbf{w}}^{T}(n \mid n)\right]
\end{gathered}
$$

Prediction state-error and its covariance as

$$
\begin{gathered}
\tilde{\mathbf{w}}(n \mid n-1)=\mathbf{w}(n)-\hat{\mathbf{w}}(n \mid n-1) \\
\mathbf{K}(n \mid n-1)=\mathrm{E}\left[\tilde{\mathbf{w}}(n \mid n-1) \tilde{\mathbf{w}}^{T}(n \mid n-1)\right]
\end{gathered}
$$

Innovation process

$$
e(n)=y(n)-\hat{y}(n \mid n-1)
$$


is the residual noise of ANC system. According to the projection theory, $\hat{\mathbf{w}}(n \mid n-1)$ is the projection of $\mathbf{w}(n)$ on a finite dimensional Hilbert space composed of $\{y(1), y(2), \cdots, y(n)\}$, thus can be given in form of a linear combination of $e(n)$

$$
\hat{\mathbf{w}}(n \mid n-1)=\sum_{k=1}^{n} \mathbf{B}_{n}(k) e(k)
$$

Where, $\left\{\mathbf{B}_{i}(k)\right\}_{k=1}^{n}$ is $L \times 1$ coefficients matrix. Based on the orthogonality of $e(n)$, we have

$$
\hat{\mathbf{w}}(n \mid n)=\hat{\mathbf{w}}(n \mid n-1)+\mathbf{G}(n) e(n)
$$

Where, $\mathbf{G}(n)$ represents the Kalman gain.

Utilizing (9) and (10) we can write residual noise as

$e(n)=\mathbf{r}_{S \lambda}(n)\left[\mathbf{F} \tilde{\mathbf{w}}(n-1 \mid n-1)+\left(\mathbf{v}_{1}(n-1)-\overline{\mathbf{v}}_{1}(n-1)\right)\right]+\left(v_{2}(n)-\bar{v}_{2}(n)\right)$

Prediction state-error covariance as

$$
\mathbf{K}(n \mid n-1)=\mathbf{F K}(n-1 \mid n-1) \mathbf{F}^{T}+\mathbf{Q}_{1}(n-1)
$$

Then the cost function of ANC system becomes

$$
\mathbf{J}_{A N C}=\mathrm{E}\left[e^{2}(n)\right]=\mathbf{r}_{S \lambda}(n) \mathbf{K}(n \mid n-1) \mathbf{r}_{S \lambda}{ }^{T}(n)+Q_{2}(n)
$$

from which we know that minimizing $\mathbf{J}$ and $\mathbf{J}_{A N C}$ are equal.

Now using (14), (18) and (20), we get the relation between $\mathbf{K}(n \mid n)$ and $\mathbf{G}(n)$

$$
\begin{aligned}
\mathbf{K}(n \mid n)= & \mathbf{K}(n \mid n-1)-\mathbf{K}(n \mid n-1) \mathbf{r}_{S \lambda}{ }^{T}(n) \mathbf{G}^{T}(n)-\mathbf{G}(n) \mathbf{r}_{S \lambda}(n) \mathbf{K}(n \mid n-1) \\
& +\mathbf{G}(n) \mathbf{r}_{S \lambda}(n) \mathbf{K}(n \mid n-1) \mathbf{r}_{S \lambda}{ }^{T}(n) \mathbf{G}^{T}(n)+\mathbf{G}(n) \mathbf{Q}_{2}(n) \mathbf{G}^{T}(n)
\end{aligned}
$$

According to the minimum principle of matrix

$$
\frac{\partial}{\partial \mathbf{G}(n)} \operatorname{tr}\{\mathrm{E}[\mathbf{K}(n \mid n)]\}=\mathbf{0}
$$

The solution of $\mathbf{G}(n)$ should be

$$
\mathbf{G}(n)=\mathbf{K}(n \mid n-1) \mathbf{r}_{S \lambda}{ }^{T}(n)\left[\mathbf{r}_{S \lambda}(n) \mathbf{K}(n \mid n-1) \mathbf{r}_{S \lambda}{ }^{T}(n)+Q_{2}(n)\right]^{-1}
$$

The Kalman filter in prediction form is

$$
\begin{aligned}
\hat{\mathbf{w}}(n \mid n)=\hat{\mathbf{w}}(n \mid n-1)+\mathbf{G}(n) e(n) \\
\hat{\mathbf{w}}(n \mid n-1)=\mathbf{F} \hat{\mathbf{w}}(n-1 \mid n-1)+\overline{\mathbf{v}}_{1}(n-1) \\
\mathbf{G}(n)=\mathbf{K}(n \mid n-1) \mathbf{r}_{S \lambda}{ }^{T}(n)\left[\mathbf{r}_{S \lambda}(n) \mathbf{K}(n \mid n-1) \mathbf{r}_{S \lambda}{ }^{T}(n)+Q_{2}(n)\right]^{-1} \\
\mathbf{K}(n \mid n-1)=\mathbf{F K}(n-1 \mid n-1) \mathbf{F}^{T}+\mathbf{Q}_{1}(n-1) \\
\mathbf{K}(n \mid n)=\left[\mathbf{I}-\mathbf{G}(n) \mathbf{r}_{S \lambda}(n)\right] \mathbf{K}(n \mid n-1)\left[\mathbf{I}-\mathbf{G}(n) \mathbf{r}_{S \lambda}(n)\right]^{T} \\
+\mathbf{G}(n) \mathbf{Q}_{2}(n-1) \mathbf{G}(n)
\end{aligned}
$$


To initialize the algorithm, set $\hat{\mathbf{w}}(0 \mid 0)=\mathbf{w}_{0}$ and $\mathbf{K}(0 \mid 0)=\mathbf{K}_{0}$. The two input signals of the Kalman Filter are $\mathbf{r}_{S \lambda}(n)$ and $e(n)$. Noted that, the form in (29) is essential in broadband noise cancellation which maintains the nonnegative definiteness of $\mathbf{K}(n \mid n)$ in order to avoid divergence.

\subsection{Implement of ANC algorithm without secondary path identification}

The measurement sensitivity matrix is filtered reference signal includes secondary path model in both measurement equation (10) and Kalman filter (25) (29). Considering ANC algorithm without secondary path identification, the measurement sensitivity matrix should be the reference signal $\mathbf{r}(n)$ because the secondary path model remains unknown, however, this violates the central premise in Kalman filter theory that the underlying state-space model is accurate, which deteriorates its performance and restrains its application. To solve this contradiction, we need to compensate the influence caused by the uncertain secondary path. It is assumed in acoustic field, that acoustic propagation in space can be simplified as amplitude decay and acoustic time delay, in case of far-field or mainly direct sound in enclosure [3]. Based on this assumption, it is reasonable to regard the dynamics of the secondary path $S(z)$ small enough to treat $\mathbf{r}_{S \lambda}(n)$ as reference signal with a small random fluctuation $\mathbf{r}(n)+\tilde{\mathbf{r}}(n)$, thus, the uncertainty of $S(z)$ is migrated to the fluctuation of $\mathbf{r}(n)$. The fluctuation of measurement sensitivity matrix is $\tilde{\mathbf{r}}(n)=[\tilde{r}(n), \tilde{r}(n-1), \ldots, \tilde{r}(n-L+1)]$, with $\mathrm{E}[\tilde{\mathbf{r}}(n)]=0$ and $\mathrm{D}[\tilde{\mathbf{r}}(n)]=\sigma_{n}, \sigma_{n}$ is $L$ order nonnegative definite matrix.

We recast the measurement equation with random fluctuation as a linear dynamic system with determined measurement matrix

$$
\begin{aligned}
\mathbf{y}(n) & =\mathbf{r}(n) \mathbf{w}(n)+\tilde{v}_{2}(n) \\
\tilde{v}_{2}(n) & =v_{2}(n)+\tilde{\mathbf{r}}(n) \mathbf{w}(n)
\end{aligned}
$$

Where $\mathrm{E}\left[\mathbf{r}_{S \lambda}(n)\right]=\mathbf{r}(n)$. The system satisfies assumptions below:

Assumption 1: $\left\{\mathbf{F}(n), \mathbf{r}_{s \lambda}(n), \mathbf{v}_{1}(n), v_{2}(n), n=0,1,2, \cdots\right\} \quad$ is a mutual independent stochastic variable sequence.

Assumption 2: $\mathbf{w}(n)$ and $\left\{\mathbf{F}, \mathbf{r}_{S \lambda}(n), n=0,1,2, \cdots\right\}$ are mutual independence. $\mathbf{w}_{0}$ and $\left\{\mathbf{v}_{1}(n), v_{2}(n), n=0,1,2, \cdots\right\}$ are mutual independence.

Assumption 3: The statistical properties of initial state $\mathbf{w}_{0}$, noises $\mathbf{v}_{1}(n)$ and $v_{2}(n), \operatorname{STM} \mathbf{F}$, measurement matrix $\mathbf{r}_{S \lambda}(n)$ are:

$$
\begin{array}{ll}
\mathrm{E}\left[\mathbf{w}_{0}\right]=\boldsymbol{\mu}_{0}, & \mathrm{E}\left[\left(\mathbf{w}_{0}-\boldsymbol{\mu}_{0}\right)\left(\mathbf{w}_{0}-\boldsymbol{\mu}_{0}\right)^{T}\right]=\mathbf{K}_{0} \\
\mathrm{E}\left[\mathbf{v}_{1}(n)\right]=\mathbf{0}, & \mathrm{E}\left[\mathbf{v}_{1}(n), \mathbf{v}_{1}(m)\right]=\mathbf{Q}_{1}(n) \delta_{n m},
\end{array}
$$




$$
\begin{array}{ll}
\mathrm{E}\left[v_{2}(n)\right]=\mathbf{0}, & \mathrm{E}\left[v_{2}(n), v_{2}(m)\right]=\mathbf{Q}_{2}(n) \delta_{n m} \\
\mathrm{E}\left[\mathbf{r}_{s \lambda}(n)\right]=\mathbf{r}(n), & \operatorname{cov}[\tilde{r}(n), \tilde{r}(n)]=C_{\tilde{r}}(n)=1
\end{array}
$$

Now we proof that (30) and (31) are in accordance with standard Kalman filter request:

i) It is easy to know

$$
\mathrm{E}\left[\tilde{v}_{2}(n)\right]=\mathrm{E}\left[v_{2}(n)+\tilde{\mathbf{r}}(n) \mathbf{w}(n)\right]=\mathbf{0}
$$

ii) Noted that $\mathbf{w}_{0}$ and $\left\{\mathbf{r}_{S \lambda}(n), v_{2}(n), n=0,1,2, \cdots\right\}$ are mutually independent, we have

$$
\mathrm{E}\left[\mathbf{w}_{0} \tilde{v}_{2}^{T}(n)\right]=\mathbf{0}
$$

iii) As for $\forall n \neq l$, because of the linear relations between $\mathbf{w}(i)$ and $\mathbf{F}^{n-l+1} \mathbf{w}_{0}$, $\mathbf{v}_{1}(l), \mathbf{F}^{i-2} \mathbf{v}_{1}(l-i)$, where $i=2,3, \cdots, l$

$$
\begin{gathered}
\mathrm{E}\left[\tilde{v}_{2}(n) \tilde{v}_{2}^{T}(m)\right]=\mathrm{E}\left[\begin{array}{l}
v_{2}(n) v_{2}{ }^{T}(m)+v_{2}(n) \mathbf{w}^{T}(m) \tilde{\mathbf{r}}^{T}(m) \\
+\tilde{\mathbf{r}}(n) \mathbf{w}(n) v_{2}{ }^{T}(m)+\tilde{\mathbf{r}}(n) \mathbf{w}(n) \mathbf{w}^{T}(m) \tilde{\mathbf{r}}^{T}(m)
\end{array}\right]=0 \\
\mathrm{E}\left[\mathbf{v}_{1}(n) \tilde{v}_{2}^{T}(m)\right]=\mathrm{E}\left[\mathbf{v}_{1}(n) v_{2}{ }^{T}(m)+\mathbf{v}_{1}(n) \mathbf{w}^{T}(m) \tilde{\mathbf{r}}^{T}(m)\right]=\mathbf{0}
\end{gathered}
$$

Define $\mathrm{E}\left[\tilde{v}_{2}(n) \tilde{v}_{2}^{T}(n)\right]=\tilde{Q}_{2}(n)$, based on the property of conditional expectation, we have

$$
\begin{aligned}
\tilde{Q}_{2}(n) & =\mathrm{E}\left[\begin{array}{l}
v_{2}(n) v_{2}(n)^{T}+v_{2}(n) \mathbf{w}^{T}(n) \tilde{\mathbf{r}}^{T}(n) \\
+\tilde{\mathbf{r}}(n) \mathbf{w}(n) v_{2}{ }^{T}(n)+\tilde{\mathbf{r}}(n) \mathbf{w}(n) \mathbf{w}^{T}(n) \tilde{\mathbf{r}}^{T}(n)
\end{array}\right] \\
& =Q_{2}(n)+\mathrm{E}\left[\tilde{\mathbf{r}}(n) \mathrm{E}\left[\mathbf{w}(n) \mathbf{w}(n)^{T}\right] \tilde{\mathbf{r}}(n)^{T}\right]
\end{aligned}
$$

Where

$$
\begin{aligned}
\mathrm{E}\left[\tilde{\mathbf{r}}(n) \mathrm{E}\left[\mathbf{w}(n) \mathbf{w}^{T}(n)\right] \tilde{\mathbf{r}}^{T}(n)\right] & =\sum_{i=0}^{L-1}\left\{\tilde{r}(n-i) w_{i}\left[\sum_{i=0}^{L-1} \tilde{r}(n-i) w_{i}\right]\right\} \\
& =\sum_{i=1}^{L} C_{\tilde{r}}(n) w_{i}{ }^{2} \\
& =\mathrm{E}\left[\mathbf{w}^{T}(n) \mathbf{w}(n)\right]
\end{aligned}
$$

Applying $\mathrm{E}\left[\mathbf{w}^{T} \mathbf{w}\right]=\mathrm{E}\left[\operatorname{trace}\left(\mathbf{w} \mathbf{w}^{T}\right)\right]=\operatorname{trace}\left(\mathbf{P}_{w w}\right)+\operatorname{trace}\left(\mathrm{E}[\mathbf{w}] \mathrm{E}\left[\mathbf{w}^{T}\right]\right)$, we get

$$
\mathrm{E}\left[\mathbf{w}^{T}(n) \mathbf{w}(n)\right]=\operatorname{tr}\left[\mathbf{K}(n \mid n-1)+\mathbf{w}(n \mid n-1) \mathbf{w}^{T}(n \mid n-1)\right]
$$

Thus, we can utilize (25) (29) in ANC algorithm without secondary path identification, replacing measurement matrix with $\mathbf{r}(n)$, attributing the fluctuation of the secondary path to measurement matrix, we can modify $\tilde{\mathbf{Q}}_{2}(n)$ by $\mathrm{E}\left[\tilde{\mathbf{r}}(n) \mathrm{E}\left[\mathbf{w}(n) \mathbf{w}^{T}(n)\right] \tilde{\mathbf{r}}^{T}(n)\right]$, to compensate the effect caused by 
secondary path, finally manage to inhibit the convergence of Klaman filter. So comes the name Model Error Compensatory Kalman Filter (MECKF).

\subsection{Determination of the model noise}

The conventional Kalman filter assumes that the statistical properties of the noise in dynamic model and observation system are exactly known, but the noise in integrated system is uncertain. One classical way to estimate noises $\mathbf{v}_{1}(n)$, $\mathbf{v}_{2}(n)$ and their statistical properties $\mathbf{Q}_{1}(n), \mathbf{Q}_{2}(n)$, is the Saga-Husa (S-H) maximum a posterior (MAP) estimator with high complexity and large computation. Here, analyzing the ANC system model, we introduce a simple and effect method to estimate $\mathbf{v}_{1}(n), \mathbf{Q}_{1}(n), \mathbf{v}_{2}(n)$ and $\mathbf{Q}_{2}(n)$.

First, consider $\mathbf{Q}_{2}(n)$, according to (4), knowing $\mathbf{v}_{2}(n)=e(n)$ easily, $\mathbf{Q}_{2}(n)$ is written as

$$
\mathbf{Q}_{2}(n)=\mathrm{E}\left[e(n) e^{T}(n)\right]
$$

Now, the measurement updating of Kalman filter is similar to that of the S-H method, by using MAP we can prove the unbiasedness of (39).

As for $\mathbf{Q}_{1}(n)$, we refer to S-H MAP, system noise is unbiased estimated by

$$
\hat{\mathbf{v}}_{1}(n)=\frac{1}{n} \sum_{i=1}^{n}[\mathbf{w}(i \mid i-1)-\mathbf{F}(n) \mathbf{w}(i-1 \mid i-2)]
$$

Noted that state approaches its optimal value iteratively from initial value, state estimation errors decrease step by step till Kalman filter converges and fluctuate around a steady-state value $\mathbf{v}_{1}(n)$. So, the estimation (40) by using state estimation errors from $n=1$ contains the biggest estimation error at the beginning, inevitably increasing $\mathbf{v}_{1}(n)$ estimation error. Thus, a more appropriate way to estimate $\mathbf{v}_{1}(n)$ is by using the latest state estimation as

$$
\hat{\mathbf{v}}_{1}(n)=\mathbf{w}(n \mid n-1)-\mathbf{F}(n) \mathbf{w}(n-1 \mid n-2)
$$

and $\hat{\mathbf{v}}_{1}(n)=\mathbf{v}_{1}(n)$ when state estimation convergences to optimal value $\mathbf{w}^{O}$. The statistical property of $\hat{\mathbf{v}}_{1}(n)$ is given by

$$
\hat{\mathbf{Q}}_{1}(n)=\mathrm{E}\left[\hat{\mathbf{v}}_{1}(n) \hat{\mathbf{v}}_{1}^{T}(n)\right]
$$

yet apparently violates the underlying assumption of Kalman filter that $\mathbf{v}_{1}(n)$ is a white noise process and $\mathbf{Q}_{1}(n)$ is a diagonal matrix. To satisfy this premise, we make $\mathbf{v}_{1}(n)$ orthogonal artificially, defining $\hat{\mathbf{Q}}_{1}(n)$ as the inner product of the system noise vector

$$
\hat{\mathbf{Q}}_{1}(n)=\operatorname{diag}\left(\hat{\mathbf{v}}_{1}(n) \cdot \hat{\mathbf{v}}_{1}(n)\right)
$$

Thus, the diagonal and non-negative definite characteristics of $\hat{\mathbf{Q}}_{1}(n)$ can be guaranteed, which reduces the amount of computation at the same time.

The advantages over S-H method given by proposed estimation (39) and (43) lay on: first, a lower computational complexity; second, avoiding subtraction in $\hat{\mathbf{Q}}_{1}(n)$ and $\hat{\mathbf{Q}}_{2}(n)$ equations guarantee non-negative definite of $\mathbf{Q}_{1}(n)$ and 
positive definite of $\mathbf{Q}_{2}(n)$ with carefree divergence; third, with no influences on the properties of $\mathbf{v}_{2}(n)$ and $\mathbf{Q}_{2}(n)$.

\section{Algorithm analysis}

In this section, we investigate computational complexity, convergence rate and the noise control effect of the proposed Model Error Compensatory Kalman Filter (MECKF) algorithm, compared with Direction Search Least Mean Square(DSLMS) algorithm [8] which has a high performance.

\subsection{Effect of measurement fluctuation}

The error of measurement matrix is

$$
\begin{aligned}
\tilde{\mathbf{r}}(n) & =\mathbf{r}_{S \lambda}(n)-\mathbf{r}(n) \\
& =\left[\sum_{i=0}^{M_{S}-1} \lambda^{-i} S_{i} \cdot r(n-i), \cdots, \sum_{i=0}^{M_{S}-1} \lambda^{-i} S_{i} \cdot r(n-L+1-i)\right] \\
& -[r(n), r(n-1), \cdots, r(n-L+1)]
\end{aligned}
$$

with its factor

$$
\tilde{r}(n)=\left(S_{0}-1\right) r(n)+\sum_{i=1}^{M_{S}-1} \lambda^{-i} S_{i} \cdot r(n-i)
$$

Based on assumptions of ANC, we model secondary path as

$$
S(n)=A_{S} \delta\left(n-k_{S}\right), k_{S}=0,1, \cdots, M_{S}-1
$$

Where $\delta(\cdot)$ represents Kronecker function. Thus (45) becomes $\tilde{r}(n)=-r(n)+\lambda^{-k_{s}} S_{k_{s}} \cdot r\left(n-k_{s}\right)$. There are two useful notification we should make: First, $\tilde{r}(n)$ would be large due to a large value of delay $k_{s}$, that is to say a long distance between secondary sensor and cancellation point, also cause the algorithm to diverge. As to the situation $r(n)$ is a periodic signal, the residual noise would be minimized with $k_{s}$ being an integral multiple of the period. Second, $\lambda$ yields a exponential forgetting effect on $\tilde{r}(n)$. Thus the algorithm will perform better with secondary path approximate to $S(n)=A_{s} \delta\left(n-k_{s}\right)$, require a small side lobe of the $S(n)$ impulse response, and narrow pass band.

\subsection{Computational complexity}

Define an iteration does not include the parameter initialization stage, our comparison uses the number of real multiplications and additions per iteration for MECKF and DSLMS algorithms in Tab.1. Note that one iteration of DSLMS defined as from direction searching stage to performance monitoring stage, and $\mathrm{N}$ is the number of sample.

From Tab.1, given the same ANC controller length $\mathrm{M}$, although, the computation complexity of MECKF is larger than that of DSLMS, this can be compromised by a faster convergence rate of MECKF compared with DSLMS, thus ensures real-time, also today DSPs can easily satisfy the computation demand. 
Besides, $M$ can be very small for MECKF with a good performance 0 , while in DSLMS, $M$ is inversely proportional to the upper bound of step size, and with $\mu$ decreasing by an order of magnitude, convergence rate decreased more than one order of magnitude, yet increasing $\mu$ for accelerating will increase the mean square residual noise, so $\mathrm{M}$ needs to balance convergence rate and noise reduction.

Tab.1 Computation complexity of MECKF and DSLMS

\begin{tabular}{ccc}
\hline Algorithm & Multiplications for one iteration & Additions for one iteration \\
\hline MECKF & $3 M^{3}+5 M^{2}+4 M+5$ & $3 M^{3}+3 M^{2}-M+1$ \\
DSLMS & $3 M+6 N+14$ & $3 M+6 N-1$ \\
\hline
\end{tabular}

\subsection{Convergence rate}

Let the original disturbance be a single-tone signal $r$ with frequency $\omega$, complex $P_{\omega}, S_{\omega}=\left|S_{\omega}\right| e^{j \theta_{\omega}}$ and $W_{\omega}(n)$ represent $P(z), S(z)$ and $W(z)$ respectively, $\left|S_{\omega}\right|$ is the amplitude of $S(z)$ and $\theta_{\omega}$ is the phase of $S(z)$. Given the one order weight updating equation as

$$
\begin{aligned}
W_{\omega}(n) & =\lambda W_{\omega}(n-1)+\lambda G(n-1) r(n-1)\left|S_{\omega}\right| e^{j \theta_{\omega}}\left[\frac{P_{\omega}}{S_{\omega}}-W_{\omega}(n-1)\right] \\
& +\lambda G(n-1) v(n-1)
\end{aligned}
$$

At the frequency where $\left|\theta_{\omega}\right|=90^{\circ}$, (47) becomes $W_{\omega}(n)=F W_{\omega}(n-1)$, $0<<<1$ will adjust weight in some sense, avoid algorithm divergence. While DSLMS will diverge in area of $\left|\theta_{\omega}\right|=90^{\circ}$ and its neighbourhood 0 .

The second term in (47) contains an instantaneous weight estimation factor $\lambda G(n-1) r(n-1)$, taking into account of state estimation error, measurement noise and reference signal power, to guide the direction of weight updating, this will accelerate convergence rate in a large scale, and MECKF is a variable step size adaptive algorithm. In DSLMS, the instantaneous weight estimation factor $\mu$ maintains constant after initialization, only its sign can be controlled during iterations.

\subsection{Noise control effect}

After convergence, the residual noise powers of MECKF and DSLMS are

$$
\begin{aligned}
& e_{\text {MECKF } \omega}(n)=r(n) P_{\omega}(1-\lambda)+\left[1-\lambda G(n-1) r(n) S_{\omega}\right] v(n) \\
& e_{\text {DSLMS } \omega}(n)=\left[1-\mu r^{2}(n) S_{\omega}\right] v(n)
\end{aligned}
$$

Though the first term in (48) contains the effect of $P_{\omega}$, the factor $\lambda$ that approximates to 1 will compromise the influence. In the second term, Kalman gain will update such that $e_{M E C K F \omega}(n)=\min$, which attenuates secondary path influences. DSLMS with a step size $\mu$ near to $0, e_{\text {DSLMS } \omega}(n)$ is mainly and greatly influenced by secondary path. Results in Part II demonstrate this point clearly. 


\section{Conclusions}

This paper deals with the design and implement of an MECKF algorithm in ANC without secondary path identification.

1) The MECKF algorithm's step size can be adapted with reference signal, state estimation error and residual noise, hence have increased convergence rate to ensure real-time.

2) The MECKF algorithm decreased the influence of the secondary path, reduced the RNP.

3) The MECKF algorithm avoided divergence in phase $90^{\circ}$ of secondary path because of the transfer matrix but improved control performance.

\section{References}

[1] P.A. Nelson , S.J.Elliott, Active Control of Sound. New York: Academic Press, 1992.

[2] S. Haykin, Adaptive Filter Theory, Prentice-Hall, Inc., 1991.

[3] S. M. Kuo and D. R. Morgan, Active Noise Control Systems, Algorithms and DSP implementations, John Wiley \& Sons, Inc., 1996.

[4] P. Lopes, M. Piedade, The Kalman filter in active noise control. in Proceedings of ACTIVE 99, Ft. Lauderdale, FL, U.S.A., December 1999; pp. 1111-1122.

[5] Fraanje Rufus, H.Sayed Ali, Verhaegen Michel, Doelman Niek J. A fast-array Kalman filter solution to active noise control. International Journal of Adaptive Control and Signal Processing. 19(2-3),pp. 125-152, 2005.

[6] T.Bambang Riyanto, R.Yacoub Redi, K. Uchida, Identification of secondary path in ANC using diagonal recurrent neural networks with EKF algorithm. 2004 5th Asian Control Conference, v 1, pp. 665-673, 2004.

[7] Chikun Gong, Hui Wang, Gen Gu, Active noise control based on adaptive inverse control with KALMAN. in Proceedings of the 8th International Symposium on Test and Measurement: Chongqing, Vol. 1(2009), pp. 547-550.

[8] D. Zhou and V. DeBrunner, A new active noise control algorithm that requires no secondary path identification based on the SPR property. IEEE Trans. Signal Processing, 55(5): 1719-1729, 2007.

[9] Yoshinobu Kajikawa, Yasuo Nomura, Active Noise Control System Without Secondary Path Model, 2000 IEEE International Symposium on Circuits and Systems: Geneva, 2000, pp. 349-352.

[10] Chengyuan Chang, Dengrui Chen, Active Noise Cancellation Without Secondary Path Identification by Using an Adaptive Genetic Algorithm, IEEE Trans. On Institution and Measurement, 59(9),pp. 2315-2327,2010.

[11] Ruihua Zhang, Zhibo Xie, Xiuli Zhang, An Improved Active Noise Control System Without Secondary Path Model. IEEE Trans. Signal Processing, 2010, pp.454-459.

[12] Halim Dunant, Li Cheng, Zhongqing Su, Virtual sensors for active noise 
control in acoustic-structural coupled enclosures using structural sensing: Robust virtual sensor design. Journal of the Acoustical Society of America, 129(3),pp. 1390-1399, 2011. 